\title{
Studies of Lead Tungstate Crystal Matrices in High Energy Beams for the CMS Electromagnetic Calorimeter at the LHC
}
G. Alexeev ${ }^{15}$,
E. Auffray ${ }^{5}$,
P. Baillon 5 ,
D. Barney5,
G. Bassompierre ${ }^{2}$,
E. Bateman ${ }^{4}$,
K.W. Bell ${ }^{4}$
Y. Benhammou ${ }^{19}$,
P. Bloch 5 ,
D. Bomestar ${ }^{18}$,
B. Borgia ${ }^{17}$,
J. Bourotte12,
S. Burge 4 ,
W. Cameron' ${ }^{8}$,
R. Chipaux 6 ,
D. Cockerill ${ }^{4}$, J. Connolly ${ }^{4}$, I. Dafinei ${ }^{5 *}$, P. Denes ${ }^{16}$, P. Depasse ${ }^{19}$, K. Deiters ${ }^{20}$,
L. Dobrzynski12, H. El Mamouni ${ }^{19}$, J. L. Faure ${ }^{6}$, M. Felcini ${ }^{21}$, M. Finger ${ }^{14}$, T. Flügel ${ }^{20}$, F. Gautheron ${ }^{2}$, A. Givernaud 6 , S. Gninenko ${ }^{11}$, N. Godinovic ${ }^{18}$, D. J. Graham ${ }^{8}$ J. P. Guillaud ${ }^{2}$ E. Guschin ${ }^{11}$, M. Haguenauer ${ }^{12}$, H. Hillemanns ${ }^{1}$, H. Hofer $^{21}$, B. Ille 19, S. Jaaskelainen ${ }^{5}$, V. Katchanov ${ }^{15}$, B. Kennedy ${ }^{4}$, T. Kirn ${ }^{1}$, M. Korzhik ${ }^{10}$, K. Lassila-Perini ${ }^{21}$, M. Lebeau ${ }^{5}$, P. Lebrun ${ }^{19}$, P. Lecoq ${ }^{5}$, G. Lecoeur ${ }^{5}$, P. Lecomte ${ }^{21}$, E. Leonardi 17, E. Locci 6 , R. Loos 5 , D. Ma13, F. Martin ${ }^{19}$, J. P. Mendiburu' ${ }^{2}$, Y. Musienko ${ }^{11}$, P. Nedelec ${ }^{2}$, F. Nessi-Tedaldi 21 , D. Newbold ${ }^{3}$, H. Newman ${ }^{13}$, M. Oukhanov ${ }^{15}$, L. Pacciani ${ }^{17}$, J. P. Peigneux ${ }^{2}$, S. Pirro ${ }^{17}$, S. Popov ${ }^{11}$, I. Puljak ${ }^{18}$, C. Purves ${ }^{3}$, D. Renker ${ }^{20}$, F. Rondeaux ${ }^{6}$, E. Rosso ${ }^{5}$, R. Rusack ${ }^{9}$, H. Rykaczewski 21, D. Schmitz ${ }^{1}$, M. Schneegans ${ }^{2}$, J. Schwenke ${ }^{1}$, C. Seez ${ }^{8}$ I. Semeniouk ${ }^{11}$, P. Shagin ${ }^{15}$, S. Shevchenko ${ }^{13}, \quad$ X. Shi ${ }^{7}$ D. Sillou ${ }^{2}$ D. Simohand ${ }^{19}$, A. Singovsky 15, $\begin{array}{ll}\text { I. Soric } & 18, \quad \text { B. Smith } \\ \end{array}$, R. Stephenson ${ }^{4}$ P. Verrecchia ${ }^{6}$ J. P. Vialle ${ }^{2}$, T. S. Virdee ${ }^{\dagger \dagger}$, R. Y. Zhu ${ }^{13}$

\begin{abstract}
Using matrices of lead tungstate crystals energy resolutions better than $0.6 \%$ at $100 \mathrm{GeV}$ have been achieved in the test beam in 1995. It has been demonstrated that a lead tungstate electromagnetic calorimeter read out by avalanche photodiodes can consistently achieve the excellent energy resolutions necessary to justify its construction in the CMS detector. The performance achieved has been understood in terms of the properties of the crystals and photodetectors.
\end{abstract}

\section{(Accepted by Nuclear Instruments and Methods)}

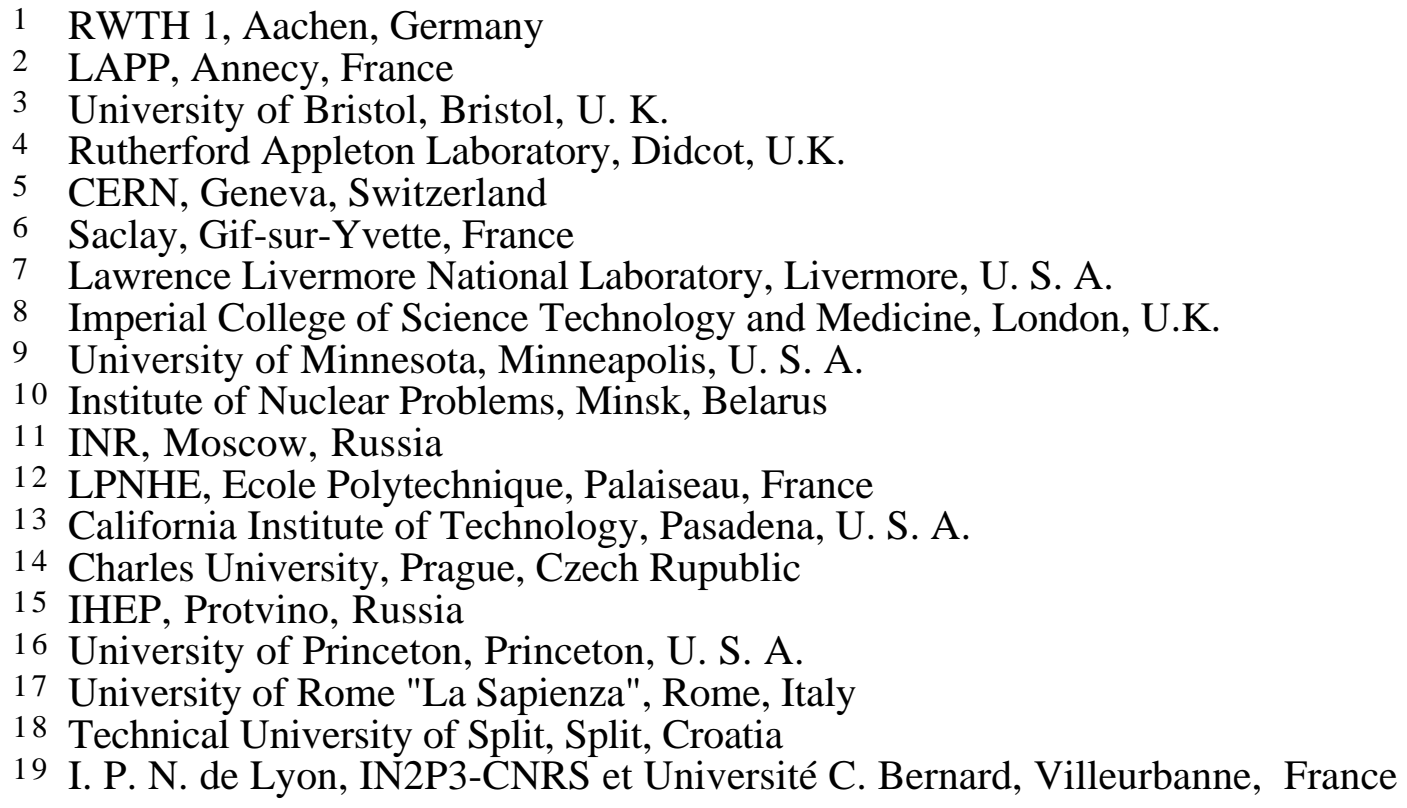


20 Paul Scherrer Institute, Villigen, Switzerland

21 ETH, Swiss Federal Institute of Technology, Zurich, Switzerland

* Now at University of Rome "La Sapienza"

$\dagger$ Also at Imperial College of Science Technology and Medicine, London 


\section{Introduction}

In Autumn 1994 the CMS collaboration chose an electromagnetic calorimeter design using lead tungstate crystals with avalanche photodiodes (APDs) as photodetectors [1]. At that time excellent resolution had only been achieved by using photomultipliers with lead tungstate crystals, although the data taken in the test beam in 1994 justified the hope that, with some modifications to the APDs and crystals, excellent resolution could also be achieved with the baseline combination [2]. In the test beam in 1995 we have consistently achieved an energy resolution of better than $0.6 \%$ at $100 \mathrm{GeV}$.

The main studies of the performance of the CMS ECAL design in 1995 were made in the H4 beamline at CERN. Table 1 lists the data-taking periods in the $\mathrm{H} 4$ beam considered in this paper.

Table 1: The data-taking periods considered

\begin{tabular}{|c|c|l|}
\hline Period & Matrix & \multicolumn{1}{c|}{ Comments } \\
\hline A: July & 18 crystals & EG\&G APDs. \\
\hline B: July/ & $\begin{array}{c}5 \times 5 \\
\text { crystals }\end{array}$ & $\begin{array}{l}\text { Central 3 x 3 equipped with two Hamamatsu APDs. } \\
\text { Comprises two periods with different } 5 \times 5 \text { matrices. }\end{array}$ \\
\hline C: September & $\begin{array}{c}7 \times 7 \\
\text { crystals }\end{array}$ & $\begin{array}{l}\text { Combined test with HCAL. Matrix of smaller lateral } \\
\text { size crystals. }\end{array}$ \\
\hline
\end{tabular}

\section{Test Setup for 1995}

The crystals used in all except the September tests were truncated pyramids with a length of $230 \mathrm{~mm}\left(25.8 \mathrm{X}_{0}\right)$ and a $20.5 \times 20.5 \mathrm{~mm}^{2}$ front face projective to a point about $1430 \mathrm{~mm}$ from the front surface, and closely approximate the crystals to be used near $|\eta|=0$ in the baseline CMS design. They were placed in a thermally insulated, double-skinned, light-tight, aluminium box on a remotely controlled moving table. The moving table was able to rotate the matrix about 2 mutually perpendicular axes, transverse to the beam, and at a distance of $1465 \mathrm{~mm}$ from the surface of the crystals. The table could also be moved horizontally, transverse to the beam, to modify the angle of incidence in one plane.

In front of the box were placed scintillation counters: two orthogonal planes of scintillation strips, $20 \mathrm{~mm}$ wide, used for adjusting the beam position, halo counters to be used offline for rejecting beam halo events, and trigger counters. The trigger and halo counter signals were all recorded in a TDC so that they could be used offline to study pileup of events. The trigger used for most of the data taking was a single coincidence defining a $20 \times 20 \mathrm{~mm}^{2}$ beam spot which corresponded well to both the beam size and the front face of a crystal. Drift chambers gave a measurement of the impact point of beam particles with an accuracy of better than $195 \mu \mathrm{m}$.

Electron beams with rates greater than $2 \mathrm{kHz}$ were available in the $\mathrm{H} 4$ beam for momenta between 15 and $150 \mathrm{GeV}$. With the momentum defining collimators closed to $\pm 3 \mathrm{~mm}$ the momentum spread of the beam is calculated to be less than $0.2 \%$. Pion contamination in the electron beam is generally at a level of $<10^{-3}$ (for example: it is found to be $\sim 5 \times 10^{-4}$ at $80 \mathrm{GeV})$.

\subsection{Temperature and Voltage}

Both the scintillation light yield of the crystal and the APD gain are temperature dependent. For the crystal the variation is $\approx-1.9 \% /{ }^{\circ} \mathrm{C}[2]$. For the APDs the fractional coefficients at a gain of 50 are of a similar magnitude, and are given in table 2 . For these reasons the temperature of the test setup had to be carefully stabilized. Inside the box the sides of the crystal matrix were in contact with water-cooled copper plates, and, during the data-taking runs discussed below the preamplifier cards were also in direct thermal contact with water-cooled copper plates. The input cooling water was maintained at $18^{\circ}, 20^{\circ}$, or $21^{\circ} \mathrm{C}$ (depending on the ambient temperature during 
the run), with a flow rate such that the exit water was less than $0.1^{\circ} \mathrm{C}$ warmer than the input water.

The temperatures of both the APDs and the crystals were continuously monitored and written to tape with the other data. The temperature monitoring of the APDs was done with small thermistor beads coupled with heat conducting paste to the rear of the APD ceramic packages. They were not calibrated before mounting resulting in an arbitrary offset on the temperature scale with an r. m. s. of about $0.7^{\circ} \mathrm{C}$. They were used to follow relative temperature changes, with a precision of about $0.02^{\circ} \mathrm{C}$. For the temperature monitoring of the crystals two types of probes were used. Platinum resistance probes were used as a known and reliable control, and successful tests were made with a small integrated circuit device that gives an output current proportional to absolute temperature ${ }^{1}$. Using the AD590 probes temperature changes could be followed with a precision of about $0.02^{\circ} \mathrm{C}$. Most crystals were instrumented with probes touching both the front and rear faces, so that a temperature map could be made. In the data-taking periods considered below, the diurnal peak-to-peak temperature fluctuation was smallest $\left(\sim 0.2^{\circ} \mathrm{C}\right)$ on the back of the crystals on the sides of the matrix, and largest $\left(\sim 0.5^{\circ} \mathrm{C}\right)$ at the front of central crystals. The temperature was also generally higher at the front and centre of the matrix, and cooler at the sides and back of the matrix. The temperature difference between the hottest and coolest part of the matrix was about $1^{\circ} \mathrm{C}$. Fig. 1 shows an example of the temperature variation measured by a temperature sensor (AD590) throughout one of the data taking periods.

The APD gain is also sensitive to applied voltage. It can be seen from table 2 that the fractional change of gain (at a gain of 50) resulting from a $1 \mathrm{~V}$ change in the applied voltage is $2 \%$ for the EG\&G APDs and 15\% for the Hamamatsu APDs. The high voltage on the APDs was monitored with precision of about $10 \mathrm{mV}$. The voltage variations were found to be insignificant.

\subsection{Readout and electronics}

Two different APDs were used for light detection, their characteristics are shown in table 2. The prime requirement for the 1995 beam tests was that the effective thickness of silicon, $d_{\text {eff }}$, and hence the response to ionizing radiation, be sufficiently small as to not give rise to the large tails seen on the high side of the energy distributions in the 1994 data [2].

Table 2: Avalanche Photodiodes used in the H4 tests in 1995

\begin{tabular}{|c|c|c|}
\hline & $\begin{array}{c}\text { EG\&G } \\
\text { C30719E }\end{array}$ & $\begin{array}{c}\text { Hamamatsu } \\
\text { S5345 } \\
\end{array}$ \\
\hline Size & $5 \times 5 \mathrm{~mm}^{2}$ & $\phi=5 \mathrm{~mm}$ \\
\hline Capacitance & 30 & 325 \\
\hline Typical operating voltage & 400 & 150 \\
\hline $\mathrm{d}_{\mathrm{eff}}(\mathrm{M}=50)$ & 7.6 & 3.5 \\
\hline Quantum efficiency at 500nm & 0.75 & 0.65 \\
\hline Excess noise factor, $\mathrm{F}(\mathrm{M}=50)$ & 2.8 & 2.0 \\
\hline $1 / \mathrm{M} \times \mathrm{dM} / \mathrm{dT}(\mathrm{M}=50) \quad\left[\%\right.$ per $\left.{ }^{\circ} \mathrm{C}\right]$ & -3.5 & -2.3 \\
\hline $1 / \mathrm{M} \times \mathrm{dM} / \mathrm{dV}(\mathrm{M}=50) \quad[\%$ per $\mathrm{V}]$ & 2 & 15 \\
\hline
\end{tabular}

The signals went into fast, low-noise pre-amplifiers designed around a high performance JFET. These amplifiers had a peaking time of $35 \mathrm{~ns}$ and measured electronics noise of about 1200 electrons when connected to an EG\&G APD and about 6000 electrons when connected to a Hamamatsu APD (referred to the preamplifier input, and measured with a charge sensitive ADC using a $200 \mathrm{~ns}$ gate). These noise performance figures imply an energy equivalent noise of

\footnotetext{
1 AD590 made by Analogue Devices.
} 
$\approx 15 \mathrm{MeV} /$ crystal with EG\&G APDs and $\approx 75 \mathrm{MeV} /$ crystal with Hamamatsu APDs.

The noise seen in the standard data taking configuration was significantly larger than this when using EG\&G APDs, for two reasons:

a) The limited dynamic range of the ADCs used ${ }^{2}$ meant that a single ADC count had a typical energy equivalent size of $30 \mathrm{MeV}$. The measured intrinsic ADC noise of nearly 1 count (i.e. somewhat larger than the $1 / \sqrt{ } 12$ of an ideal ADC) thus made a significant additional contribution to the measured noise.

b) Coherent pick-up noise on the 80 metre cable from the test area to the control room, although insignificant on a single channel, added about $30 \%$ to the width of the sum of nine channels used to reconstruct the incident energy. This figure was achieved after the insertion of fast pulse transformers in the signal lines to isolate the beam area ground from the ADC ground - without the pulse transformers the coherent noise added about $60 \%$ to the width of a nine channel sum.

Thus in the standard data taking configuration the energy equivalent of noise measured for a sum of nine crystals was about $130 \mathrm{MeV}$ with the EG\&G APDs, and $200 \mathrm{MeV}$ with the Hamamatsu APDs.

During some of the tests two APDs were mounted on some of the crystals. In this case each APD was followed by a complete and independent electronics chain, and read out in a separate ADC channel.

A relatively large slow component was present in the scintillation of the crystals used in 1995. This slow component had not been present in earlier crystal samples, and has been eliminated from more recent crystal samples [3]. For this reason the data used in the analysis presented below were taken using an ADC gate of $300 \mathrm{~ns}$. Data were also taken with other gate lengths. With a $200 \mathrm{~ns}$ gate the charge collected decreased by between 20 and $30 \%$. This includes the effects of the preamplifier shaping and the dispersion in $80 \mathrm{~m}$ of RG58 cable.

\subsection{Monitoring the calibration}

The calibration was monitored using short $(\approx 30 \mathrm{~ns})$ pulses of light transported to the rear of each crystal by optical fibres. The light pulses were generated by a cluster of red LEDs ${ }^{3}$. The electronics generating the pulses used continuous feedback of the signal size, via a Si photodiode, to stabilize the pulse size. No instability of the pulse size was measurable, implying that the stability better than $0.1 \%$.

\subsection{Crystal preparation}

Many of the crystals used in the 1995 tests were cut from ingots of insufficient diameter and so had sizable chamfers on their long edges $(\sim 2-3 \mathrm{~mm})$. In addition the cutting was done by hand resulting in both lateral size variations, and aplanarity of the sides $(\approx 0.5 \mathrm{~mm})$. Thus the study of lateral uniformity and resolution close to crystal boundaries was delayed until the final period when geometrically better (although still hand cut) crystals became available.

On delivery to CERN crystals are inspected by eye and measured geometrically before being given an extensive series of quality checks, including tests of light yield, of optical absorption as a function of wavelength, and of longitudinal uniformity.

The crystals used in the tests were wrapped in Tyvek 4 on all sides except the rear, where the APDs were mounted. When using the EG\&G APDs much of the remaining rear crystal surface was also covered with Tyvek. This was not possible when two APDs were mounted on each crystal, as was done with Hamamatsu APDs. Comparing the results it seems that the additional

${ }^{2}$ Charge integrating LeCroy 1881 ADCs, 64 channels in a single Fastbus module.

${ }^{3}$ Stanley Super Bright SBR5501, peak emission at $660 \mathrm{~nm}$.

${ }^{4}$ Tyvek is a trademark of Dupont. We used type 1056, with a nominal thickness of $160 \mu \mathrm{m}$. 
Tyvek on the rear surface resulted in an increase of $\sim 20 \%$ in the light collected.

\section{Energy Resolution}

Data taking for energy resolution studies was generally preceded by calibration. Using a $50 \mathrm{GeV}$ electron beam, runs of $40 \mathrm{k}$ events were taken with the $20 \times 20 \mathrm{~mm}^{2}$ trigger counter aligned with each of the towers in turn. This was then followed by an energy scan — runs of 100k events were taken with the beam momentum set at each of 7 momenta in turn $(15,25,35,50,80$, 120 and $150 \mathrm{GeV}$ ) using the same trigger counter aligned on each of the towers to be studied, in turn.

\subsection{Analysis procedure}

Analysis of an electron run is begun by locating, in terms of the drift chamber coordinates, the centre of the crystal covered by the trigger. This is done by finding the point of maximum signal in that crystal. This procedure is reproducible with a precision of better than $0.1 \mathrm{~mm}$. A calibration is made with the $50 \mathrm{GeV}$ runs, by assuming that for electrons incident close to the centre of the crystal $40 \mathrm{GeV}$ is deposited in the crystal. This gives a precise relative calibration between crystals and a rough absolute scale. Our calibration is intended to be used to reconstruct energy by summing 9 crystals, and about $80 \%$ of the energy deposited in the 9 crystals is in the central crystal.

In practice the observed signal value is obtained by fitting a Gaussian function to the peak of the spectrum seen for electrons incident in a $4 \times 4 \mathrm{~mm}^{2}$ region centred on the crystal centre. The precise absolute scale used in the plots is obtained by requiring that the reconstructed energy is equal to the beam momentum.

The energy is reconstructed by summing $3 \times 3$ crystals centred on the struck crystal, see Fig. 2. Notice that the large tails seen on the high side of the energy distributions in the 1994 data [2], due to the large APD response to ionizing radiation, are only slightly visible with the EG\&G APD this year, and not visible at all when using the Hamamatsu APD. For the results presented in this section only events where the electron is incident in a $4 \times 4 \mathrm{~mm}^{2}$ region in the centre of the $3 \times 3$ array are used. This largely excludes any effects resulting from the large gaps between badly cut crystals, and calibration errors. It also excludes the need to correct for the varying fraction of average lateral leakage out of the $3 \times 3$ array.

In these studies no cuts were applied to the data apart from requiring a well reconstructed track in the drift chambers. This cut excludes events where no track has been found because of chamber inefficiency, but it also tends to cut double particles, and some close in time pileup (the drift time is $420 \mathrm{~ns}$ ). Cuts against pileup using the halo and trigger counters were not used in the analysis reported here so as to have a simple single analysis procedure applicable to all data during half of one period the TDC was not read out. Pileup effects were studied by examining the pedestal events taken randomly during the spill, as well as by examining the pileup TDC. Pileup effects were found in the data, and understood to be due to a long overshoot tail on the signal pulse caused by the signal coupling via isolation pulse transformers. The magnitude of this tail was small, $\approx 0.5 \%$ of the pulse peak, but its length, $\approx 150 \mu \mathrm{s}$, resulted in an observable distortion of the in-spill pedestals see Fig. 3.

In addition there is a small shift in the mean value of the pedestals taken inside the spill, when compared to those taken outside the spill. This shift, which can be seen in Fig. 3, is believed to be caused by charge build up when using the ADC in a "virtual ground" configuration.

The noise term of the energy resolution was calculated run by run. The in-spill pedestals were summed using the same calibration as for the beam events. The width of this distribution was measured by a Gaussian fit to a region $\pm 1.5 \sigma$ about the peak. This width was found to be generally between 5\% and 10\% larger than the width of the out of spill pedestal.

The noise was subtracted quadratically from the energy resolutions before fitting to obtain 
the constant and stochastic terms (see Fig. 4). As a test, after subtracting the noise, a noise term was left as a free parameter in the fit. A value consistent with zero was found for this parameter. When, however, the pedestals taken between spills were tried as a measure of the noise and this procedure repeated, the fit gave a small positive value to the free noise parameter.

\subsection{Summary of results obtained}

In period A there were 4 crystals that could be used as centres of sums of 9 crystals. In period B there were 9 such crystals, and each of these crystals had 2 APDs. One can consider the resolution using the first APD, the second APD, or both APDs together. For each of these cases (there are 40 for single APDs and 18 for double APDs, plus a few which were measured more than once) the procedure outlined above was followed through. The results, for single APDs are given in table 3 .

Table 3: Summary of energy resolution results (single APD)

\begin{tabular}{|c|c|c|}
\hline & Stochastic term & Constant term \\
\hline A & $\approx 4.5 \% / \sqrt{\mathrm{E}}$ & $\approx 0.40 \%$ \\
(4 values: see Fig. 10) & & $\approx 0.35 \%$ \\
\hline B (1 (st run $)^{(18 \text { values })}$ & $\approx 4.25 \% / \sqrt{\mathrm{E}}$ & $0.30-0.71 \%$ \\
\hline B (2 (nd run) $^{\text {(18 values) }}$ & $\approx 4.00 \% / \sqrt{\mathrm{E}}$ & $\approx 0.45 \%$ \\
\hline
\end{tabular}

The statistical errors on these values are negligible - typically about $0.1 \%$ for the stochastic term and $0.02 \%$ for the constant term. The most serious systematic uncertainty is the way in which the magnitude of the noise subtracted affects the sharing of the resolution between the stochastic and constant terms in the fit. For example: reducing the noise subtracted by $10 \%$ typically results in an increase in the fitted stochastic term by slightly more than $0.5(\%)$ and a decrease in the fitted constant term by slightly less than $0.05(\%)$. Such an error in the measured noise could, for example, be caused by unusual pileup conditions. The range of values given in the table is somewhat misleading because the extremes seem to be dominated by this sort of problem. The typical values given are a more reliable guide. Results using the signals from two APDs on the central crystal improved the stochastic term of the resolution function by an amount consistent with the hypothesis that doubling the APD coverage doubled the amount of light collected. Taking all the available 18 pairs of values the mean value of the improvement factor is 1.8 , although the distribution is rather wide (r. m. s. of 0.3). The two APD results were obtained by using two APDs on the central crystal only, so if doubling the APD coverage resulted in a doubling of the light collected one would have expected an improvement factor of about 1.8.

\subsection{Stochastic term and light yield}

Monte Carlo shower simulation predicts a stochastic term for energy reconstruction in a sum of nine crystals of about $2 \% / \sqrt{ } \mathrm{E}$ (due to lateral leakage). The larger stochastic term observed in our crystals can be explained by photostatistics. Here we compare the measurements of light collected in the APDs using muons, and using the width of an LED pulse signal, with the values calculated from the stochastic term. In this section, and in the one that follows and discusses the constant term, we make the assumption that both the constant and stochastic terms are dominated by the characteristics of the central crystal, since about $80 \%$ of the energy deposited in the $3 \times 3$ array is contained in the central crystal.

Data taken with a $225 \mathrm{GeV}$ muon beam provide us with a useful measure of the light yield as collected by the APDs. Muons passing through the APDs induce a direct signal as well as the signal from scintillation light caused by the energy they deposit in the crystal. Thus one can pick 
out the sensitive area of the APD by selecting events with large energy deposits. Fig. 5 shows the impact point of muons depositing more than $400 \mathrm{MeV}$. Selecting on the impact point one can obtain two peaks: one from muons passing only through the crystal giving only the light signal, and one from muons also passing through the APD and giving light plus charge. An example is shown in Fig. 6. The light yield, as seen by the APD, can then be calculated for each crystal. The peaks of the distributions shown in Fig. 6 are fitted, and it is assumed that the most probable direct muon signal is 95 electrons per $\mu \mathrm{m}$ of effective Si thickness. It is assumed that the most probable energy deposited in the crystals by the muon is $285 \mathrm{MeV}$, and the effective thickness of the APDs is as in table 2. These assumptions, taken together, probably have a total uncertainty of about $30 \%$.

Another measure of the light yield as collected by the APDs is provided by the LED monitoring system. The light yield can be calculated assuming that the width of the observed LED signal, after subtraction of noise, is due to photostatistics. The excess noise factor is assumed to be that given in table 2. In Fig. 7 the result of this calculation is plotted against the result coming from muon runs, for each of the 9 central crystals, each with 2 APDs, in the August period. The strong correlation suggests that the relative precision (channel to channel) of these results is better than 5\%, although in view of the uncertainties in the absolute scale, the fact that the points lie on the slope $=1$ line in the plot may be fortuitous.

To obtain the crystal light yield from these numbers it is necessary to assume a matching factor for the light collected into the APDs, and a quantum efficiency. Making reasonable assumptions: a matching factor equal to the area matching (i.e. 1/23 for EG\&G, and 1/29 for Hamamatsu), and quantum efficiencies as given in table 2, leads to values around $70 \gamma / \mathrm{MeV}$, similar to those obtained by other measurements.

The light yield as calculated by these methods can then be compared with what would be calculated from the stochastic terms of the fitted energy resolutions, after subtracting the nonphotostatistics contribution which shower simulation shows to be $\approx 2 \% / \sqrt{ } \mathrm{E}$. Fig. 8 shows the results. In only three cases is the correlation poor (the point marked with a triangle is, in fact, off scale). Close examination of the cases with poor correlation shows that, in each case, the resolution function fit has produced a very small stochastic term and a very large constant term.

\subsection{Constant term and longitudinal uniformity}

The longitudinal uniformity of light collection has been measured using a low momentum $(405 \mathrm{MeV}$, kinetic energy $=84 \mathrm{MeV})$ proton beam at the Paul Scherrer Institute. The crystal is set up with its long axis perpendicular to the beam direction, and the signal in a photomultiplier tube, covering the rear face of the crystal, is recorded as a function of the longitudinal coordinate of the impinging protons. Fig. 9 shows the result of some measurements, giving the response as a function of distance down the length of the crystal, for the 4 central crystals in the period A matrix. The longitudinal uniformity measurements have been used in a shower simulation Monte Carlo. It is found that the increase in response towards the back of the crystal can compensate for rear leakage and eliminate the tail on the low energy side otherwise seen in shower simulations neglecting this non-uniformity. Severe non-uniformity, however, increases the width of the observed energy distributions and induces a constant term. In particular it is observed that the region around shower maximum must be relatively flat to avoid inducing a sizable constant term. Comparing the Monte Carlo predictions with the test beam data provides good agreement to the extent that the few crystals predicted by the Monte Carlo to generate large constant terms are indeed measured to have large constant terms. It should be noted that the longitudinal response is measured with a photomultiplier tube covering the whole rear face of the crystal, whereas the APDs cover less than $1 / 20$ of the rear face. The photomultiplier also has a rather different spectral response to the APDs. Both these factors probably mean that the longitudinal responses measured may not be quite the same as those seen by the APDs.

Fig. 10 shows the energy resolution as a function of energy for the sums of 9 centred on the 
4 towers in the centre of the period A matrix. The Monte Carlo predicts that the longitudinal nonuniformity will contribute $0.62 \%$ and $0.41 \%$, respectively, to the constant terms for energy sums centred on crystals 1045 and 1042, and make a small contributions, $0.06 \%$ and $0.20 \%$ to sums centred on 1050 and 1051. The observed energy resolutions match this prediction reasonably well. Fig. 11 shows, for the period B matrix, the predicted contribution to the constant term coming from longitudinal non-uniformity versus the fitted value after subtracting $0.3 \%$ quadratically from the fitted value. The constant terms obtained when using each of the 2 APDs are very similar, and, for this plot the mean of the 2 values has been taken. The $0.3 \%$ subtracted from the measured constant term represents an observed floor in the values found. The constant term predicted by a simulation of shower energy deposition gives a constant term of about $0.2 \%$ coming from leakage.

\section{Lateral Uniformity}

Much of period $\mathrm{C}$ was devoted to a combined test with a hadron calorimeter prototype, but high statistics electron beam data were also taken in a series of runs scanning horizontally across the matrix, to provide a first look at the lateral uniformity of a matrix. In this test the matrix was tilted at $3^{\circ}$ to the beam direction in the horizontal plane (i.e. the same angle of tilt as in the baseline design of the CMS calorimeter). The crystals used in this test had a smaller lateral size $\left(18 \times 18 \mathrm{~mm}^{2}\right.$ front face) than those used in the other tests. They were better cut and there were only small chamfers on the long edges, but still suffered from lateral size variations and aplanarity of the sides.

The horizontal scan was made across the 3 central row of crystals, using the $20 \times 20 \mathrm{~mm}^{2}$ trigger counter with runs scanning across the matrix in steps of half a crystal width, to provide adequate overlap between successive table positions. This was done at three beam momenta.

Fig. 12 shows the mean energy reconstructed in a sum of nine crystals, as a function of the impact point across four crystals, using data taken with an incident beam momentum of $50 \mathrm{GeV}$. The vertical spread of the impact points has been limited to $\pm 1 \mathrm{~mm}$. The most obvious feature is the apparent dip in the response in the centre of each crystal. A possible explanation is that there is an enhancement of light collection when light is generated close to the crystal sides, although this effect has not, until now, been seen in light ray tracing simulations.

\section{Charged Pion Rejection}

A number of high statistics charged pion runs were taken, allowing a study of the rejection power of the electromagnetic calorimeter against charged pions faking electrons. Fig. 13 shows the summed energy observed in a $3 \times 3$ array of crystals when a) electrons, and b) pions, both with a momentum of $80 \mathrm{GeV}$, are incident uniformly over the surface of the central crystal. Among the 80,000 pion events taken there are very few where the apparent energy deposited in the ECAL is larger than the beam momentum. This is in contrast to the case in 1994 where the large response to ionising radiation of the APDs used resulted in a significant tail of events with a reconstructed energy larger than beam momentum. It can be seen that a cut on reconstructed energy provides a large rejection factor against pions. Such a cut would correspond, in the CMS experiment, to matching the track momentum to the reconstructed energy in the ECAL.

A further cut, on the lateral shower shape, can be used to obtain more rejection. This cut is made on the matching of the track position with the position found in the calorimeter. The calculated position in the calorimeter depends upon the shower lateral profile. The cut is illustrated in Fig. 14. The S-cut variable is the calculated shower centre-of-gravity, in one of the two transverse dimensions, for particles incident within a particular $1 \times 1 \mathrm{~mm}^{2}$ pixel. A related cut, taking the sum rather than the difference of the rows of crystals adjacent to the central one, provides some further rejection power. Fig. 15 shows the rejection power as a function of electron efficiency when the E/p and lateral shape cuts are combined. It can be seen that a rejection greater than 5000 can be obtained with an electron efficiency of $90 \%$. 
The rejection measured here is not the same as that which would be achieved in a complete CMS detector where the momentum measurement error on the charged track would also play a role. Moreover electrons will radiate in the material of the tracker. This contributes to the momentum measurement error, but can also provide an identifying signature.

\section{Conclusion}

We have demonstrated that a lead tungstate electromagnetic calorimeter read out by avalanche photodiodes can consistently achieve the excellent energy resolutions necessary to justify its construction for the CMS detector. The performance achieved, and the small differences in performance when the beam is incident on different crystals, have been understood in terms of the properties of the crystals and APDs.

These measurements have enabled us to define more precisely the requirements for the avalanche photodiode and its associated electronics, and those we expect to use in 1996 will approximate very closely to a final version. We have begun to determine the detailed requirements on the crystals and on the associated mechanical and optical structures.

\section{Acknowledgments}

We would like to thank C. Ercoli of CERN for her help with the analysis of the temperature sensor data. We would also like to thank all the engineers and technicians from the collaborating laboratories who have helped with the work described in this paper. We also grateful for the cooperation of other members of the CMS collaboration, and in particular those of the CMS ECAL group.

\section{References}

[1] CMS, the Compact Muon Solenoid, Technical Proposal, CERN/LHCC 94-38, LHCC/P1, (15 December 1994)

[2] J. P. Peigneux et al., Results from tests on matrices of lead tungstate crystals using high energy beams, Nucl. Instr. and Meth. A378 (1996) 410

[3] M. Nikl et al., Slow components in the photoluminescence and scintillation decays of $\mathrm{PbWO}_{4}$ single crystals, Phys. Stat. Sol. 195 (1996) 311 


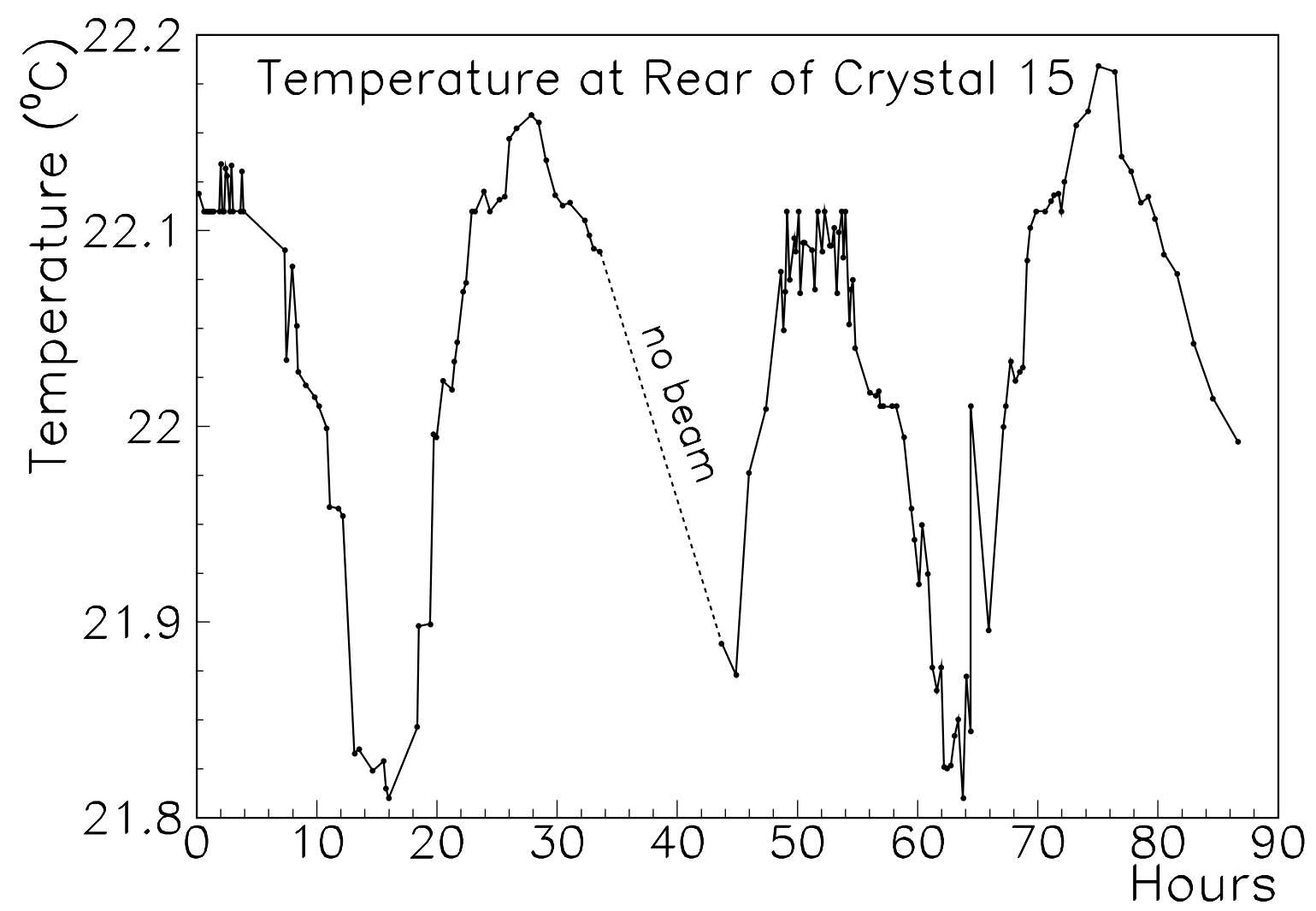

Fig. 1: Temperature measured by the AD590 temperature sensor on the rear face of a crystal during period $\mathrm{B}$ 

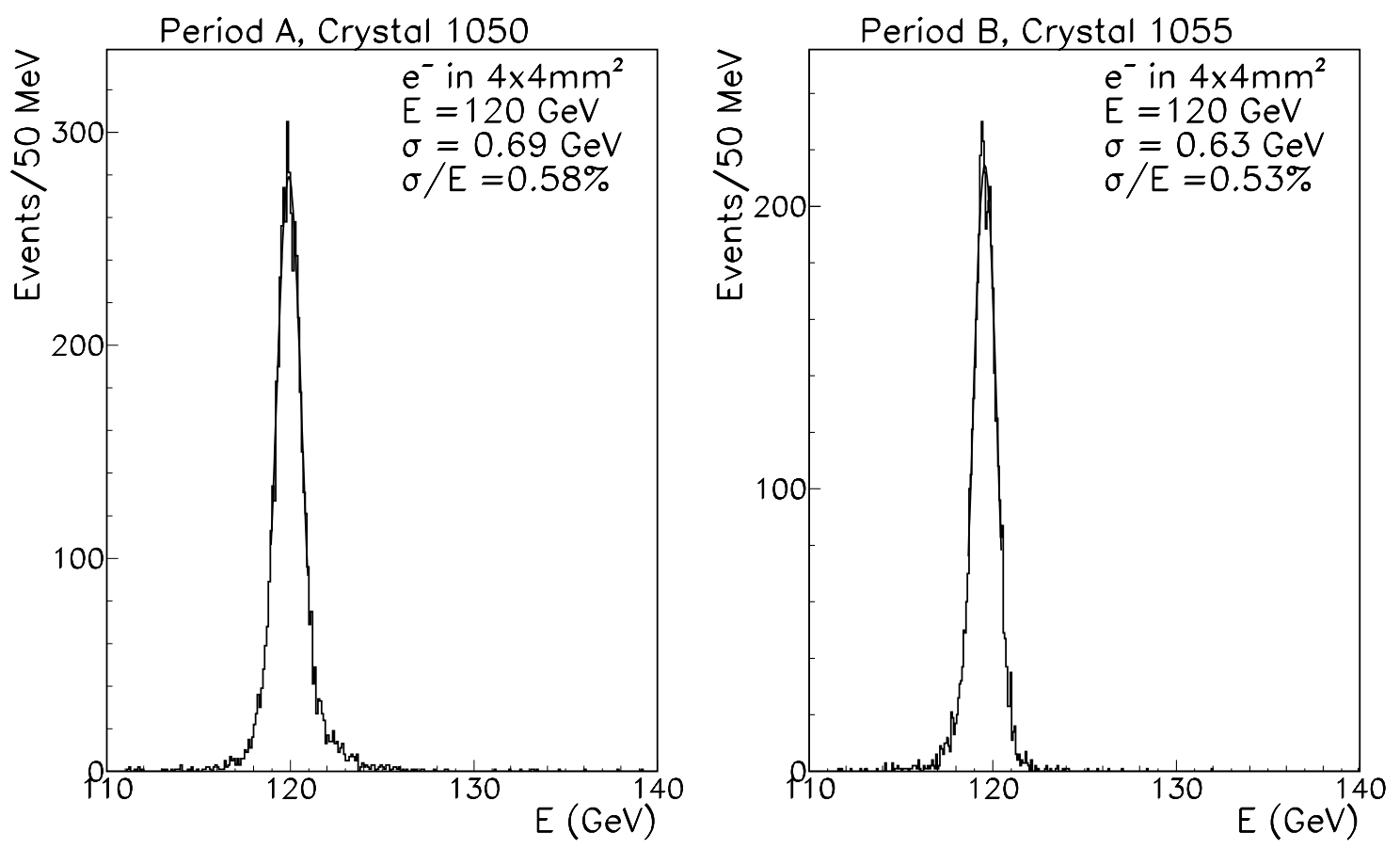

Fig. 2: Energy summed in a $3 \times 3$ array of crystals for $120 \mathrm{GeV}$ electrons incident in a $4 \times 4 \mathrm{~mm}^{2}$ region about the centre of the central crystal. Spectra from two different data taking periods are shown, the first uses EG\&G APDs, the second uses Hamamatsu APDs.

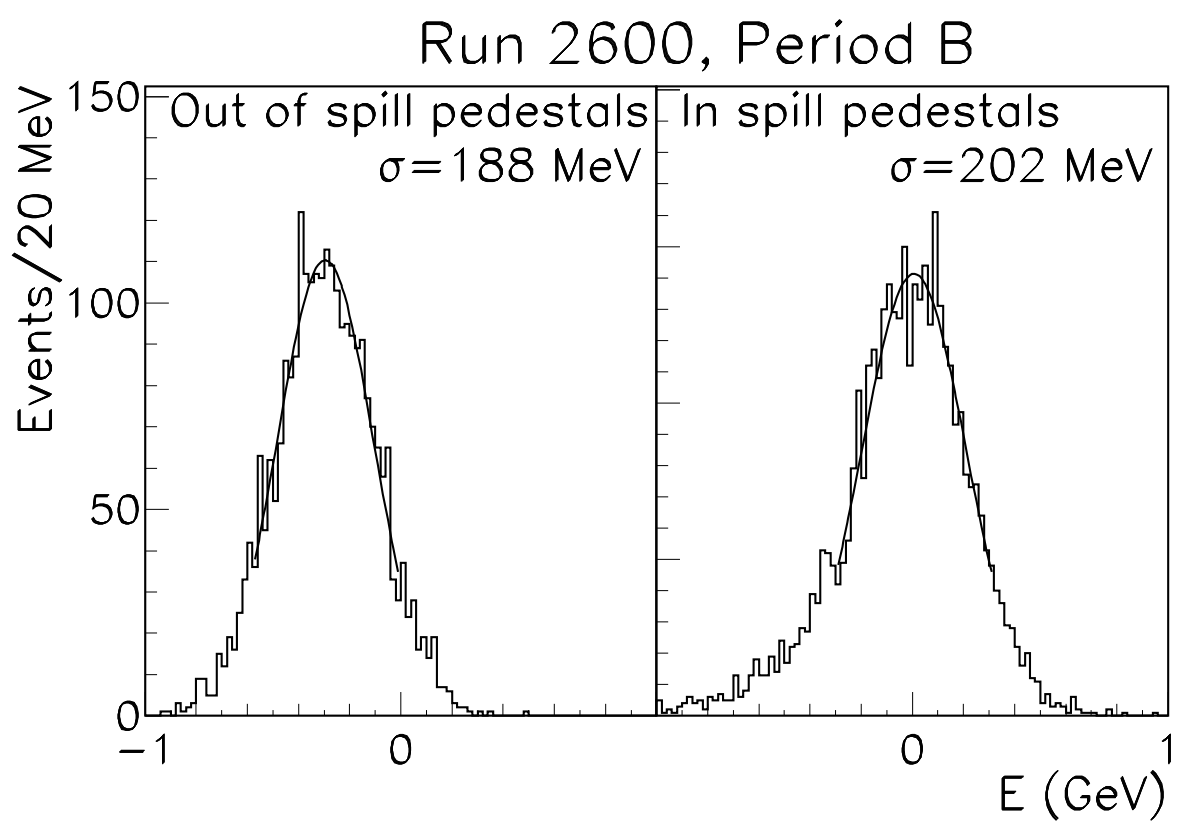

Fig. 3: Energy summed in a $3 \times 3$ array of crystals for randomly timed triggers during a $50 \mathrm{GeV}$ electron run. The triggers used for the histogram on the left are taken outside the beam spill, and those used in the histogram on the right are taken inside the spill. The zero point on the energy scale has been calculated using the in spill pedestals. 


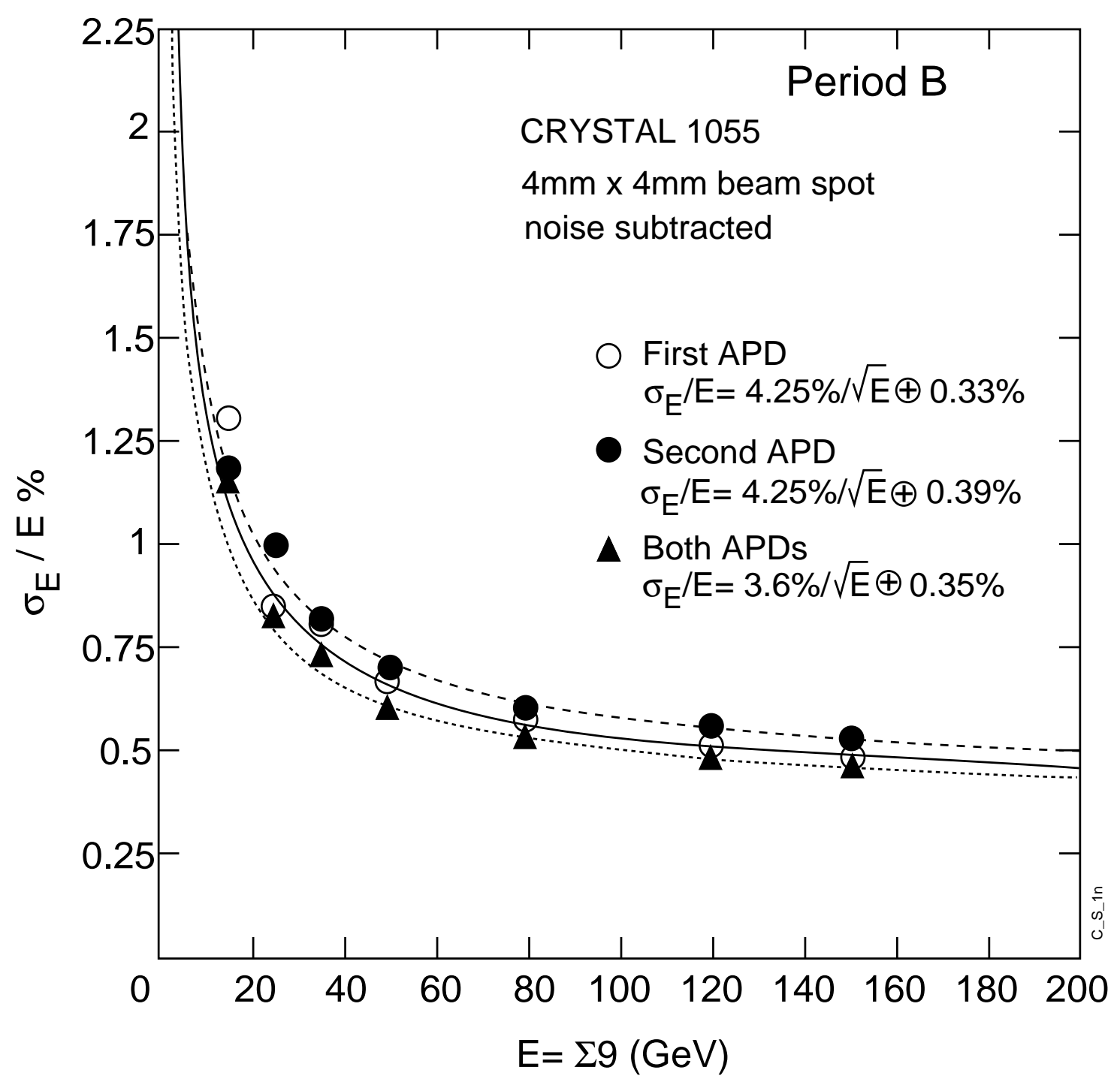

Fig. 4: Energy resolution as a function of beam energy for electrons incident in a $4 \times 4 \mathrm{~mm}^{2}$ region in the centre of the $3 \times 3$ array centred on crystal 1055 , during period $\mathrm{B}$ 


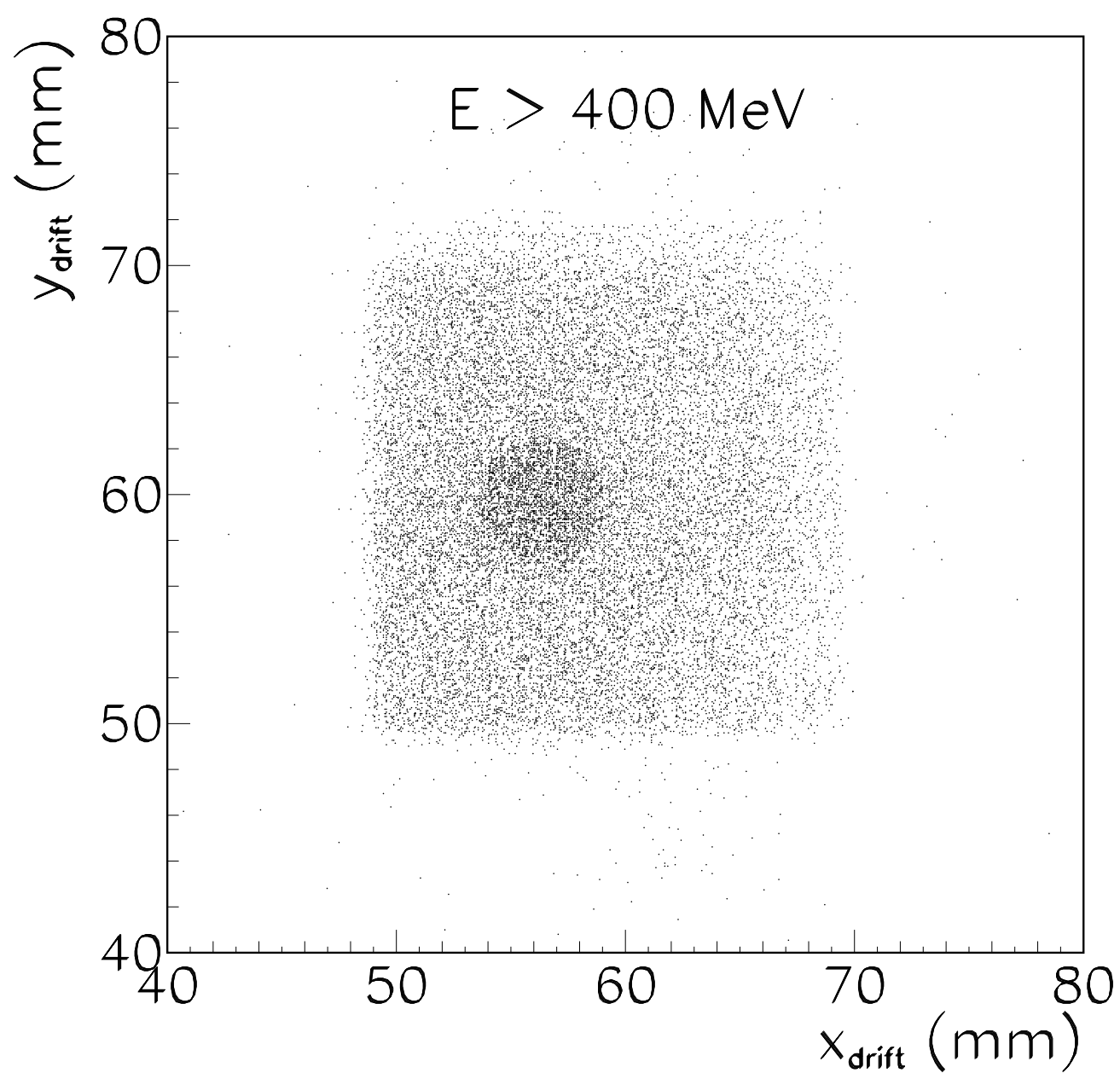

Fig. 5: Impact point of muons giving a signal of greater than $400 \mathrm{MeV}$. 


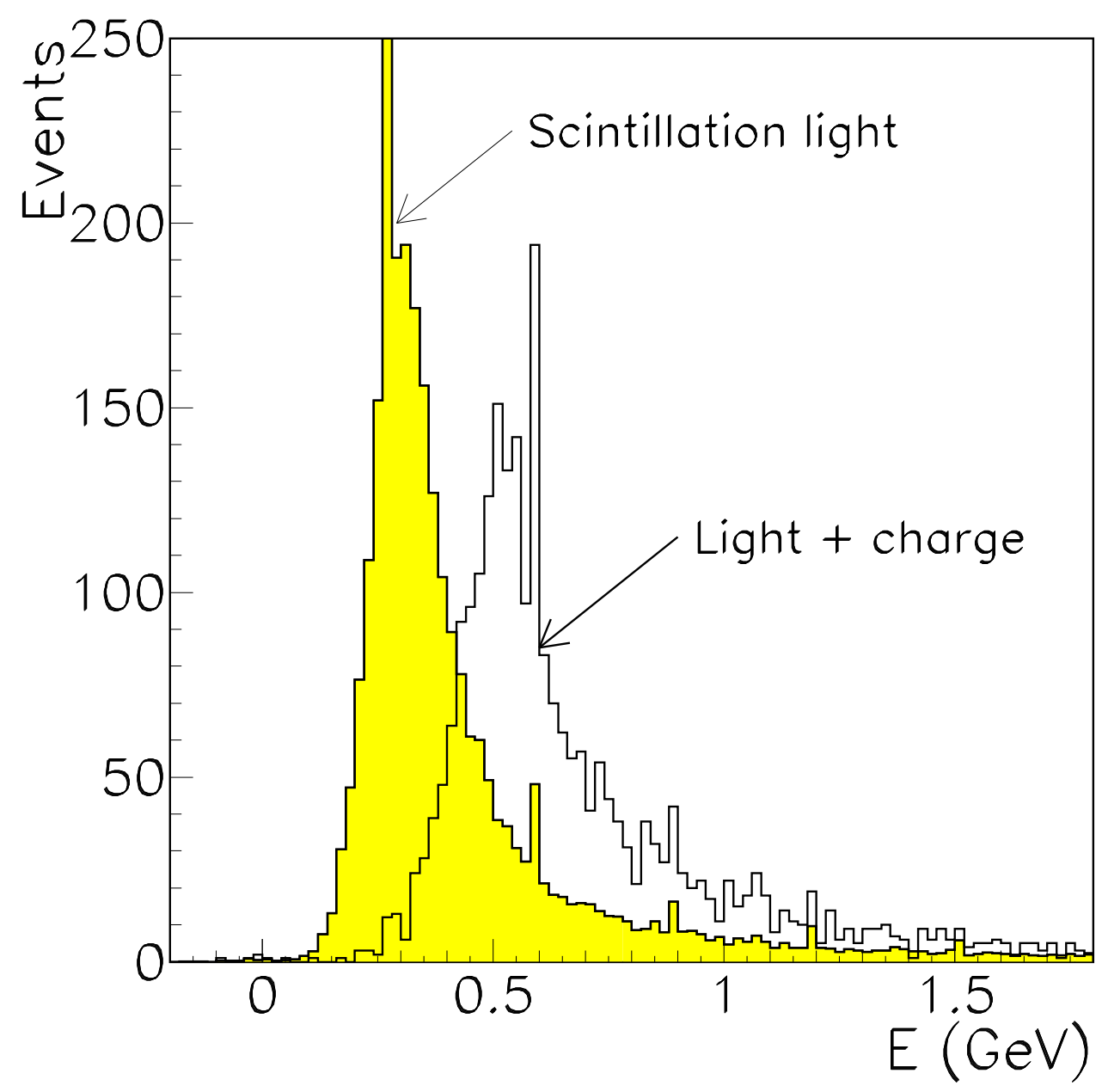

Fig. 6: Signals seen from muons passing through the crystal only (shaded histogram) and from muons passing through both crystal and APD. 


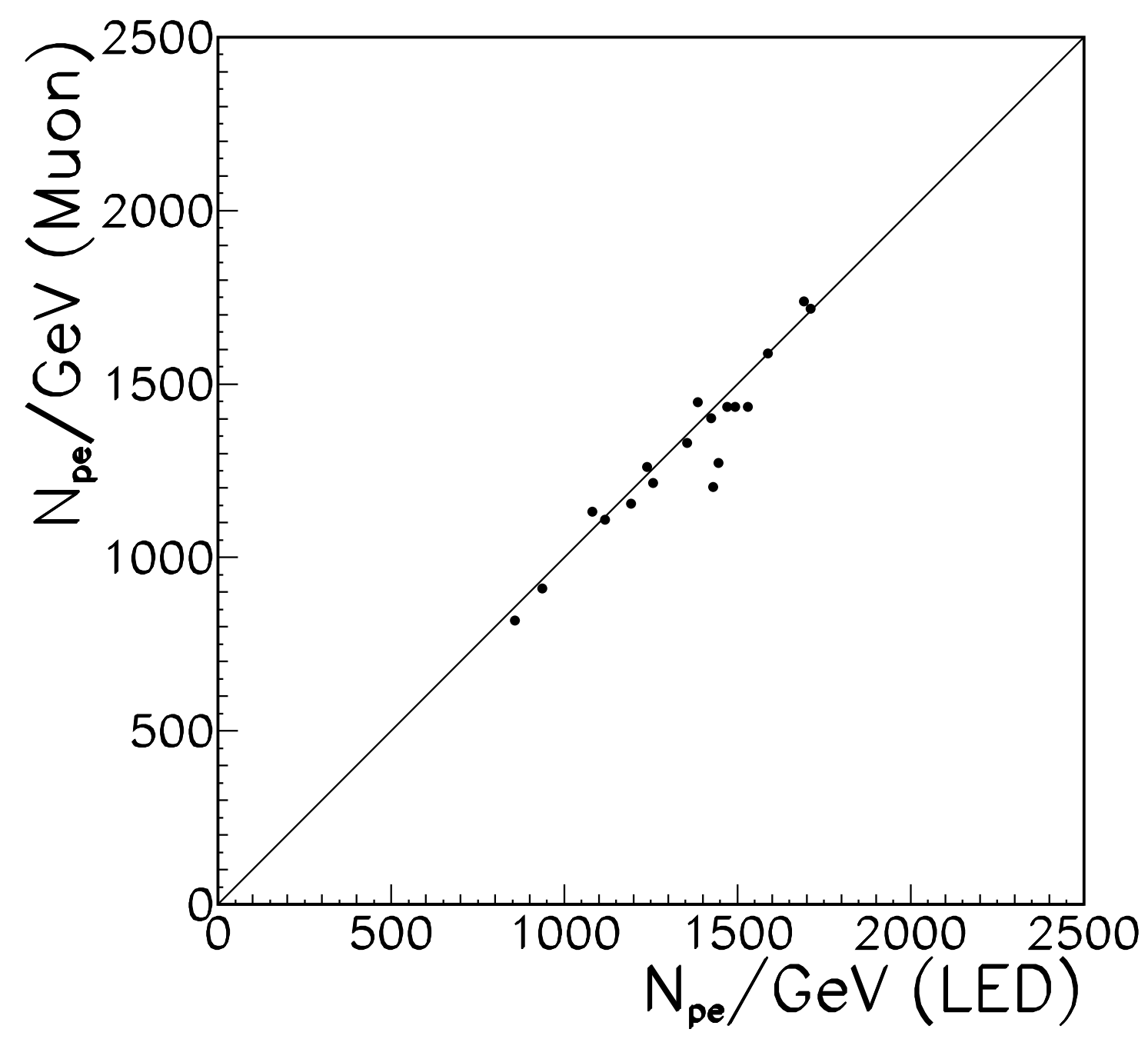

Fig. 7: Photoelectron yield in 18 APDs of the August matrix as calculated from LED signals compared to photoelectron yield as calculated from the muon signal. The line of unity gradient is drawn to help the eye. 


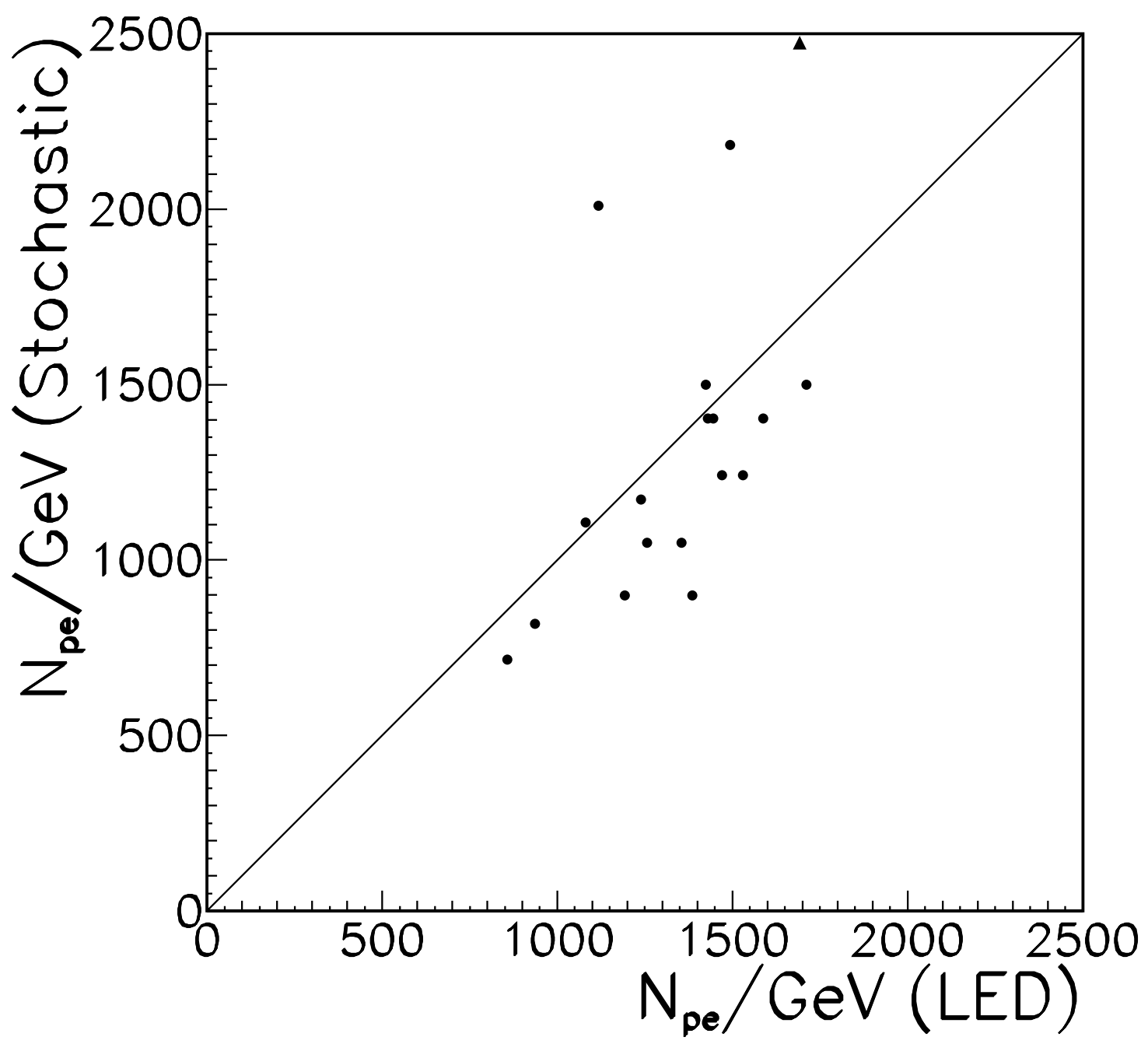

Fig. 8: Photoelectron yield in 18 APDs of the August matrix as calculated from LED signals compared to photoelectron yield as calculated from the stochastic term of the measured energy resolution function. The line of unity gradient is drawn to help the eye. 

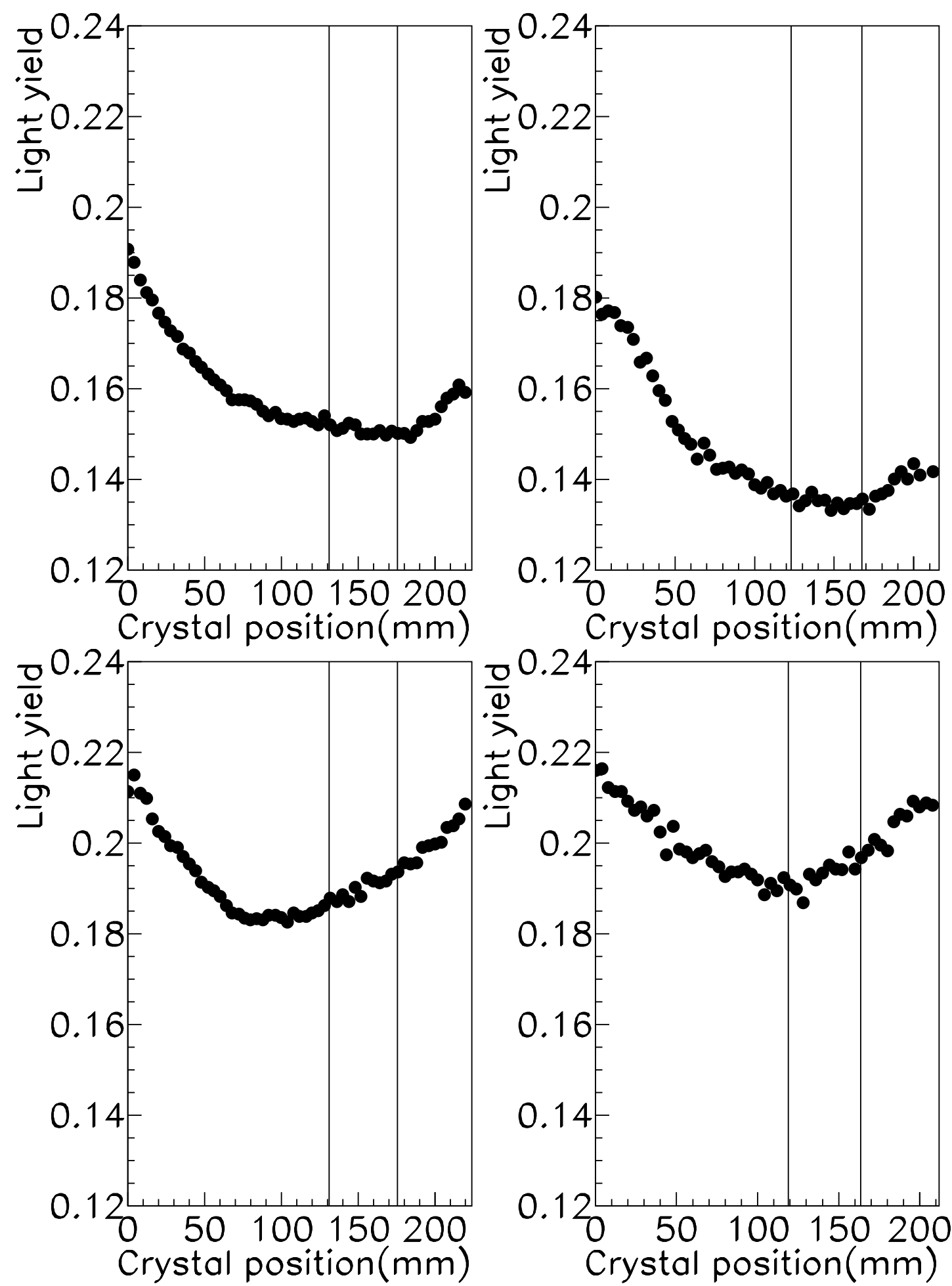

Fig. 9: Collected light as a function of longitudinal point of generation, for the four central crystals of the period A matrix, as measured in a low energy proton beam $(\mathrm{p}=405 \mathrm{MeV})$ at PSI. The horizontal scale measures the distance of the point of incidence from the back of the crystal. The vertical scale is arbitrary. The vertical lines indicate depths of 5 and 10 radiation lengths from the front of the crystal. 


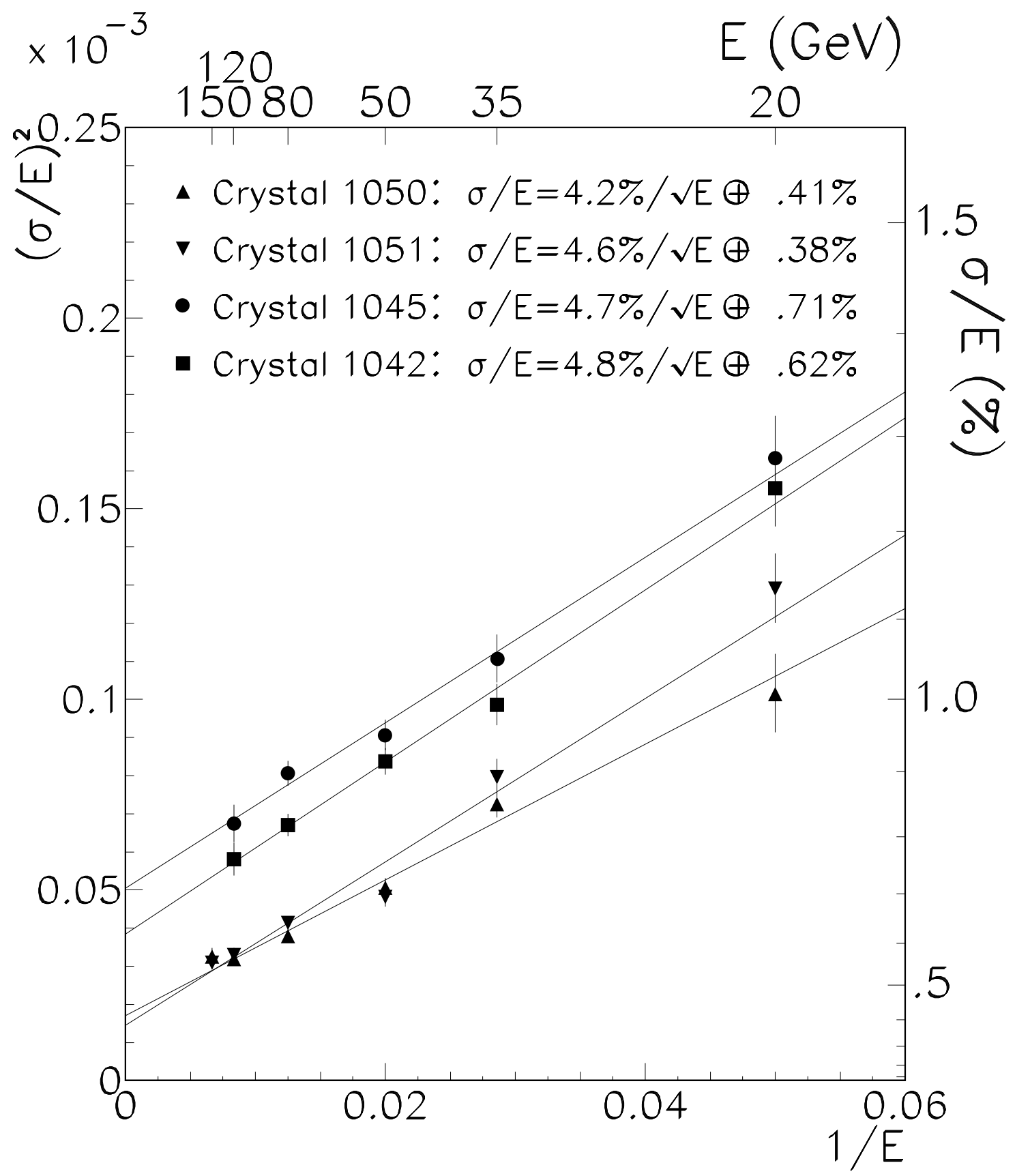

Fig. 10: Energy resolution (after noise subtraction) as a function of energy for the 4 central towers in period A. 


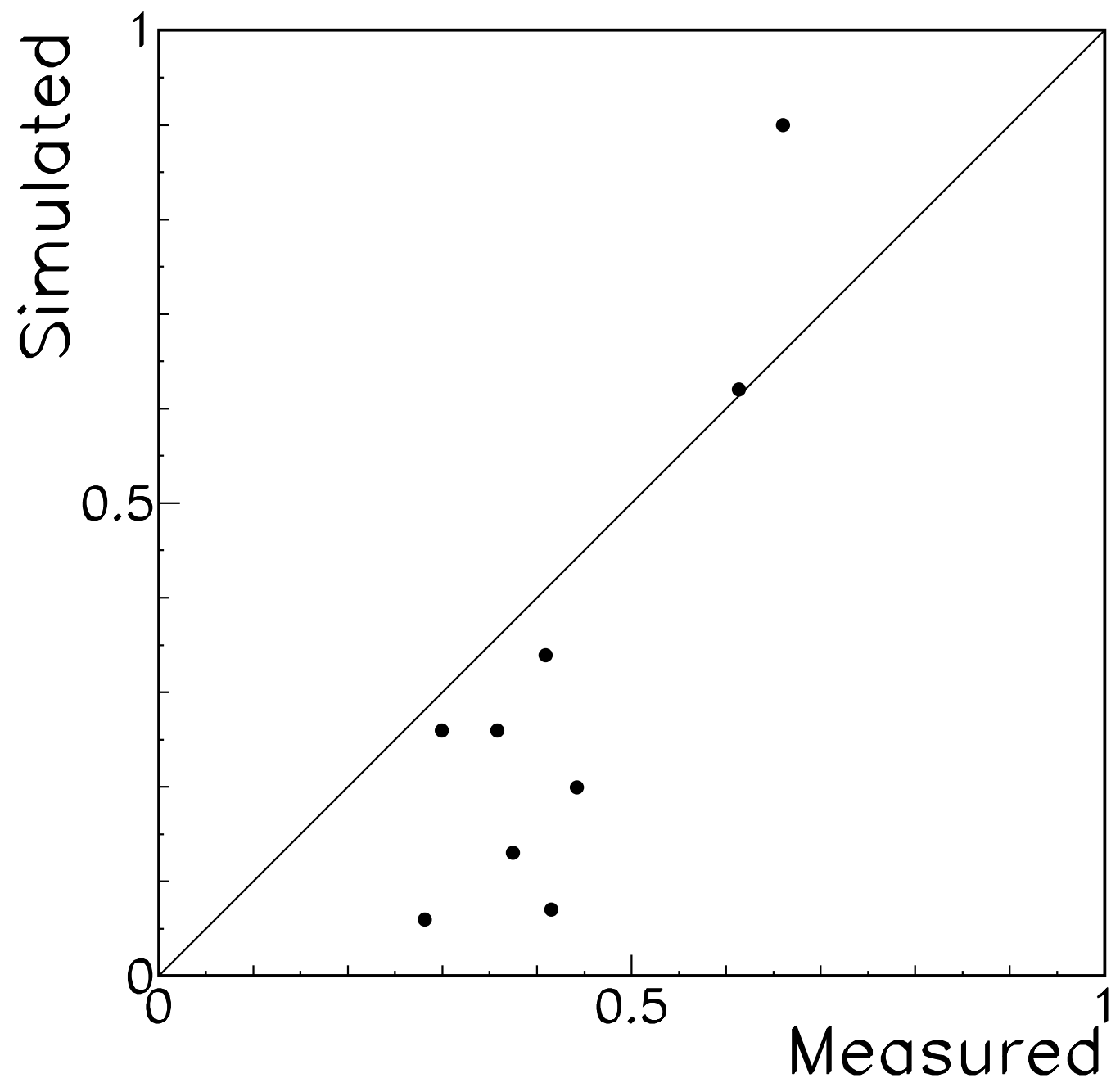

Fig. 11: Constant term of the parameterized energy resolutions for event sets centred on the 9 central towers of the period B matrix (after subtracting $0.3 \%$ quadratically), compared to the contribution to the constant term from longitudinal non-uniformity of light collection as predicted by simulation. The line of unity gradient is drawn to help the eye. 


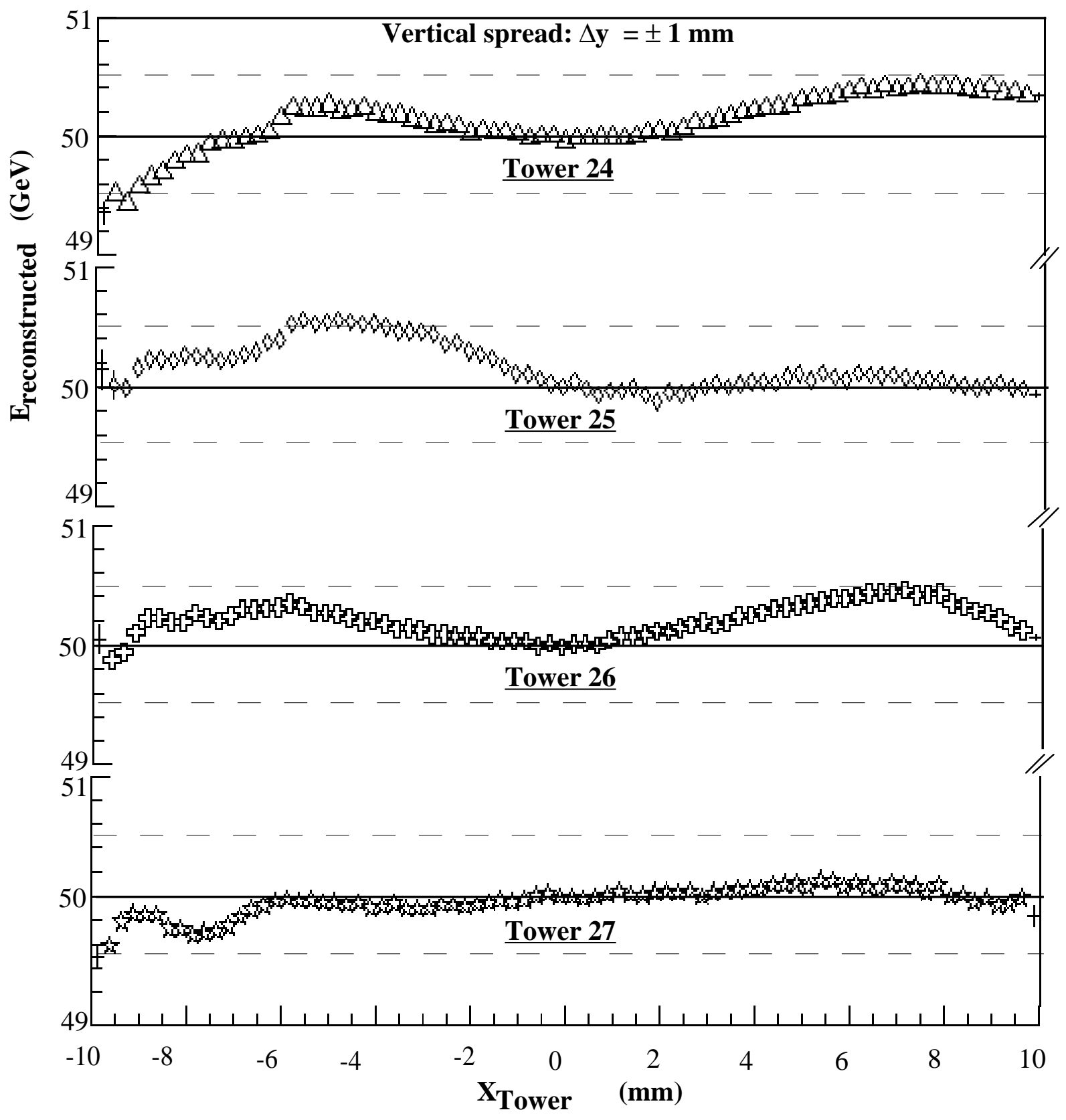

Fig. 12: Reconstructed energy as a function of position in 4 adjacent towers 

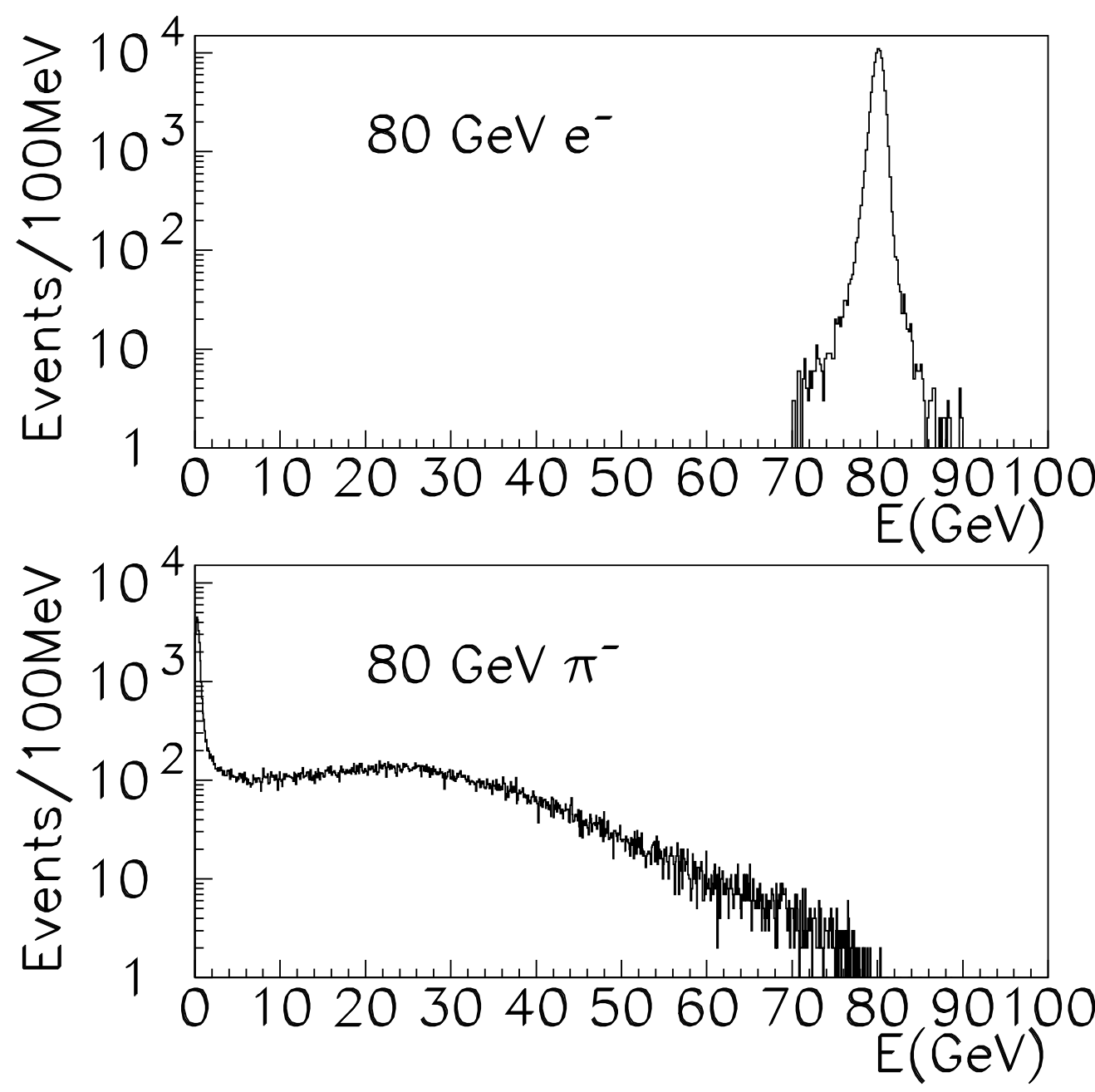

Fig. 13: Reconstructed energy for $80 \mathrm{GeV}$ electrons (top) and pions (bottom) 


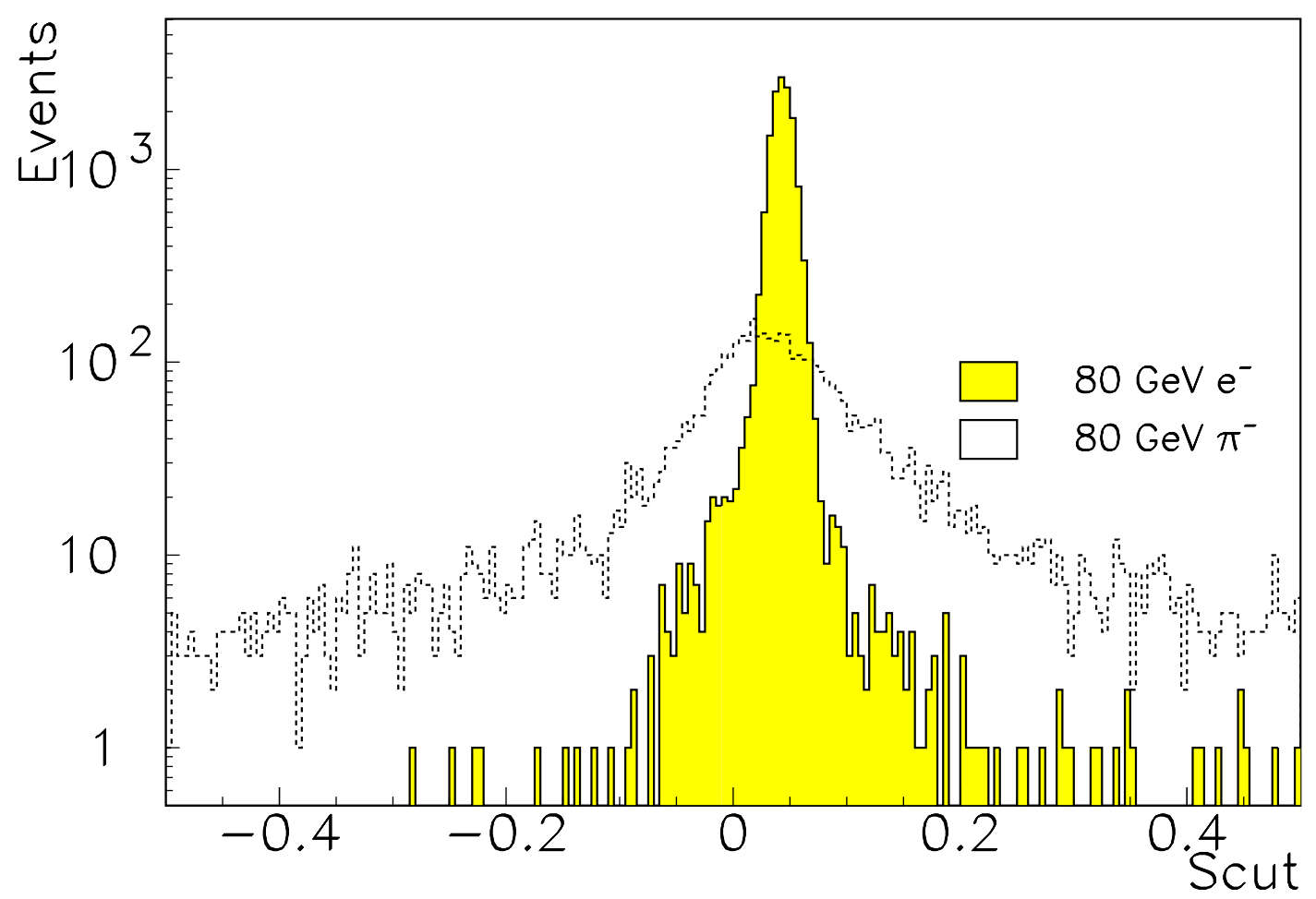

Fig. 14: Lateral shape variable (see text) for $80 \mathrm{GeV}$ electrons (shaded histogram) and pions (dashed histogram)

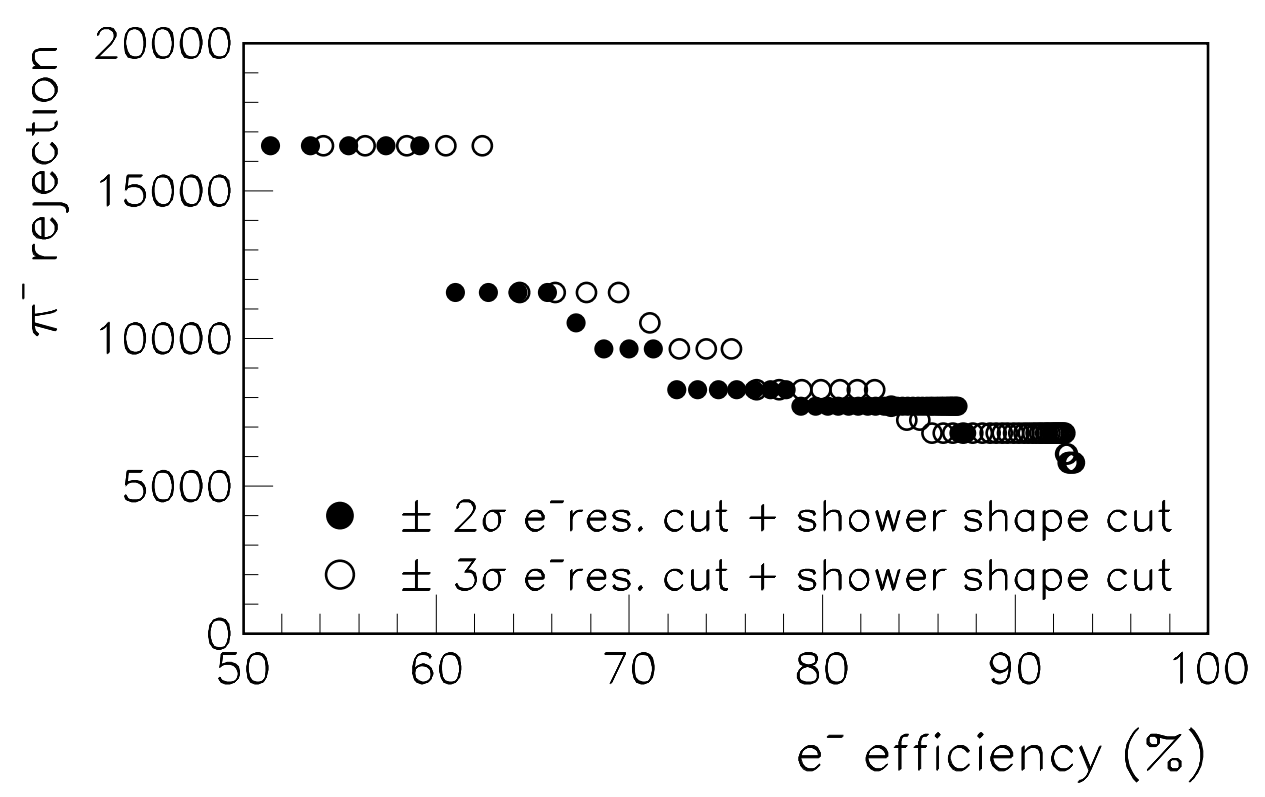

Fig. 15: Charged pion rejection factor as a function of electron efficiency at $80 \mathrm{GeV}$ 